\title{
Nonlinear relationship between multimodal adrenergic responses and local dendritic activity in primary sensory cortices
}

\author{
Yair Deitcher ${ }^{1}$, Yonatan Leibner ${ }^{1}$, Sara Kutzkel ${ }^{2}$, Neta Zylbermann ${ }^{1}$, and Michael \\ London ${ }^{1,2}$ \\ ${ }^{1}$ The Edmond and Lily Safra Center for Brain Sciences (ELSC) and the ${ }^{2}$ Life Sciences \\ Institute, The Hebrew University of Jerusalem, Jerusalem, 9190401, Israel.
}

\section{Abstract}

The axonal projections of the adrenergic system to the neocortex, originating from the locus coeruleus (LC), form a dense network. These axons release the neuromodulator norepinephrine (NE) which is involved in many cognitive functions such as attention, arousal, and working memory. Using two-photon $\mathrm{Ca}^{2+}$ imaging of $\mathrm{NE}$ axons in the cortex of awake mice, we investigated what drives their phasic activity. We discovered that NE axons in the primary somatosensory cortex responded robustly and reliably to somatosensory stimulation. Surprisingly, the same axons also responded to stimuli of other modalities (auditory and visual). Similar responses to all three modalities were observed in the primary visual cortex as well. These results indicate that phasic responses of NE axons to sensory stimuli provide a robust multimodal signal. However, despite the robustness, we also noticed consistent variations in the data. For example, responses to whisker stimulations were larger than to auditory and visual stimulations in both the barrel and the visual cortices. To test whether the variations in NE axonal responses can carry behaviorally meaningful information, we trained mice in an associative auditory fear conditioning paradigm. We found that following conditioning the response of $\mathrm{NE}$ axons increased only for $\mathrm{CS}+$, namely the signal undergoes experience-dependent plasticity and is specific to meaningful sounds. To test if variations in NE axonal responses can differentially affect the cortical microcircuit, we used dual-color two-photon $\mathrm{Ca}^{2+}$ imaging and studied the relationship between the activity of NE axons and local dendrites. We found dendritic $\mathrm{Ca}^{2+}$ signals in barrel cortex in response to auditory stimuli, but these responses were variable and unreliable. Strikingly, the probability of such dendritic signals increased nonlinearly with the $\mathrm{Ca}^{2+}$ signals of NE axons. Our results demonstrate that the phasic activity of the noradrenergic neurons may serve as a robust multimodal and plastic signal in sensory cortices. Furthermore, the variations in the NE axonal activity carry behaviorally meaningful signals and can predict the probability of local dendritic $\mathrm{Ca}^{2+}$ events. 


\section{Keywords}

40 Norepinephrine, dendritic computation, sensory cortices, locus coeruleus, cortical microcircuit, axonal two-photon imaging

\section{Introduction}

Neuromodulation plays an important role in cortical processing and sensory integration. A dominant neuromodulator in the cerebral cortex is norepinephrine (NE) secreted by neurons of the locus coeruleus (LC), a small brain stem nucleus comprised of only $\sim 1,500$ neurons in rodents (Swanson 1976). LC neurons fire tonically and their firing rate correlate with brain states. LC neurons also show phasic evoked activity patterns in response to salient stimuli. These LC neurons are active in various conditions such as in sleep-wake transitions (Aston-Jones and Bloom 1981b; Carter et al. 2010), during specific aspects of learning (Sara and Segal 1991), and during novel sensory stimulation (Hervé-Minvielle and Sara 1995). LC neurons project widely all over the brain, including to the cerebral cortex. However, it is not clear if they form a homogeneous population which projects to all cortical regions or perhaps form subpopulations with specific projection patterns. Experimentally there are conflicting results. On the one hand, tracing methods found that the LC is a relatively homogeneous structure in which neurons receive similar inputs from many sources and send output broadly to the similar brain regions (Schwarz et al. 2015; Kim et al. 2016). On the other hand, other studies showed heterogenous organization of the LC demonstrating selective projection neurons to distinct cortical areas (Chandler et al. 2014; Waterhouse and Chandler 2016). Debate continues about the homogenous or heterogeneous nature of LC axons, and the specificity of their projections to the cerebral cortex, which may have significant functional consequences.

NE has been shown to modulate sensory processing in multiple ways, including selective gating, improvement of signal-to-noise ratio and sharpening of neurons tuning curves (Berridge and Waterhouse 2003; Manunta and Edeline 2004; Sara 2009; Polack et al. 2013). The main method for deducing NE activity is to record the spiking activity of LC neurons (Aston-Jones and Bloom 1981b). However, NE-release from axons in the cortex might not scale linearly, or even monotonically with the firing rate of LC neurons (Florin-Lechner et al. 1996). Furthermore, only a sub-population of LC neurons project to the neocortex (Schwarz et al. 2015; Kim et al. 2016; Rho et al. 2018), thus recording from LC neurons is not sufficient for understanding the activity of NE fibers in sensory cortices. 
To detect in situ NE secretion, several studies used in vivo electrochemical measurements of NE. However, these methods still suffer from limitations such as the time scale of voltammetric detection and selectivity to NE (Park et al. 2018). Recent studies used two-photon $\mathrm{Ca}^{2+}$ imaging to directly measure activity of NE axons in the cortex and to correlate their activity with pupil diameter and locomotion (Reimer et al. 2016; Breton-Provencher and Sur 2018). Others have developed optical imaging methods to measure the binding of NE to various fluorescent indicators (Muller et al. 2014; Dunn et al. 2018; Feng et al. 2019). However, little is known about the sensory cues that induce phasic NE-release in sensory cortex and the consequences of this release on synaptic integration in dendrites and spines, where the adrenergic receptors are found (Herkenham 1987; Nicholas et al. 1993; Aoki et al. 1998; Wang et al. 2007).

85 Here, we examined the conditions leading to evoked responses of NE axons in two sensory cortices. Using two-photon $\mathrm{Ca}^{2+}$ imaging in awake mice, we found that NE axons in the barrel and visual cortices responded to stimuli of various modalities, including somatosensory, auditory and visual. We further show that the NE axonal responses to auditory stimulation in the barrel cortex exhibit associative plasticity, following acquisition of associative auditory fear conditioning. Finally, to examine directly the relationship between the axonal activity of NE projections and cortical dendritic activity, we performed dual-color two-photon $\mathrm{Ca}^{2+}$ imaging of local dendrites and NE axons. Interestingly, we found that local dendrites in the barrel cortex responded to auditory stimulation, and the probability of these events increases monotonically with the activity levels of NE axons. Our results are consistent with the hypothesis that NE can tune the excitability and sensitivity of dendrites to long range input projections (Phillips et al. 2016, 2018; Labarrera et al. 2018) .

\section{Results}

100 Response of $\mathrm{Ca}^{2+}$ activity in LC axons to whisker stimulation in the barrel cortex

To examine the distribution of norepinephrine (NE) axons in the barrel cortex, we used selective immunostaining for the noradrenaline transporter (NET), which is expressed exclusively in noradrenergic axons (Lorang et al. 1994). We found dense axonal innervation throughout the neocortical layers in the barrel cortex (Figure 1A). To image

105 the activity of adrenergic axons in the barrel cortex, we selectively expressed the genetically encoded $\mathrm{Ca}^{2+}$ indicator GCaMP6s (Chen et al. 2013) in the Locus Coeruleus (LC) of knock-in mice expressing Cre in tyrosine hydroxylase positive neurons (Lindeberg et al. 2004, Figure 1B). We validated the specificity of viral 
expression by comparing fluorescence in the LC and in the barrel cortex, with that of tyrosine hydroxylase (TH) and NET immunofluorescence, respectively (Figure 1C). Indeed, all axons expressing GCaMP6s were labeled with the NET antibody $(1,770$ regions of interest (ROIs), 3 mice).

We then imaged the adrenergic fibers in the barrel cortex in head-fixed awake mice 115 (Figure 1D). In agreement with previous reports (Reimer et al. 2016; BretonProvencher and Sur 2018), we found a correlation between the activity of NE axons and pupil diameter (Figure S1A-B, $n=16$ ROIs, 4 Fields of View (FoV), 3 mice).

As sensory evoked response in NE axons have never been explored, we tested whether in the barrel cortex the NE axons would respond to air puff stimulation applied to the whiskers. We found evoked responses to whisker stimulation with a prominent increase in $\mathrm{Ca}^{2+}$ (Figure 1E-G, mean evoked response (for definition see Methods): $24.39 \% \pm 1.25 \%$, bootstrap: $1.9 \pm 0.13 \% ; n=78$ ROIs, 1,777 trials, 15 FoV, 4 mice; $p$ $<0.001, t$ test). We validated that these responses were reflecting real signals and were not due to movements of the imaging plane by comparing the axonal responses

125 to the change in signal over time of auto-fluorescent 'blebs' (Reimer et al. 2016, Figure S1C, axonal evoked response, $24.39 \% \pm 1.25 \%$, blebs evoked response $0.92 \pm 0.33 \%$, $\mathrm{n}=78$ axonal ROIs, $\mathrm{n}=59$ blebs ROIs, 15 FoV, 4 mice; $\mathrm{p}<0.001$, $\mathrm{t}$ test).

The axonal response appeared in single trials of stimulation (Figure $1 \mathrm{H}$ ), with a response probability of $80 \%$, indicating a reliable axonal response (Figure 1 , response probability: $0.8 \pm 0.02$; bootstrap: $0.3 \pm 0.01 ; \mathrm{n}=78 \mathrm{ROIs}, 1,777$ trials, $15 \mathrm{FoV}, 4$ mice; $p<0.001, t$ test. Figure S1D, response probability axons: $0.8 \pm 0.02$; blebs: $0.09 \pm$ $0.01 ; n=78$ axonal ROls, $n=59$ blebs ROls, 15 FoV, 4 mice; $p<0.001$, $t$ test). We conclude that NE axons in barrel cortex display a robust and reliable response to whisker stimulations which can be detected even in individual trials. 


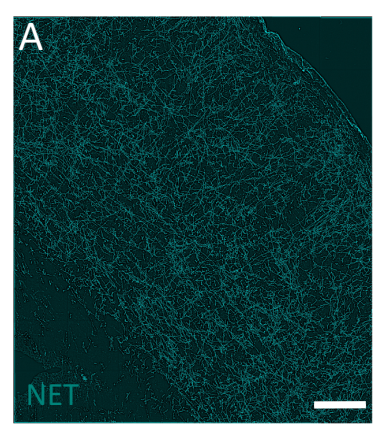

\section{B}

TH-IRES-Cre

Flex-GCaMP6s

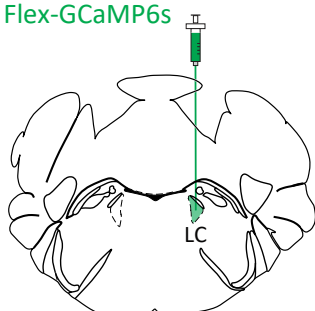

$E$

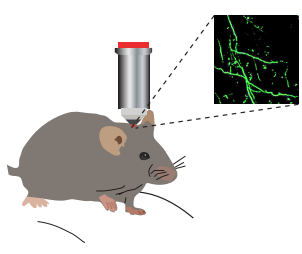

G

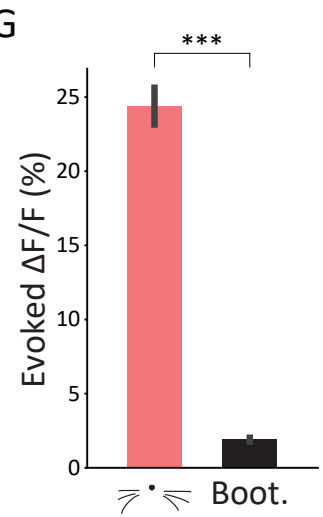

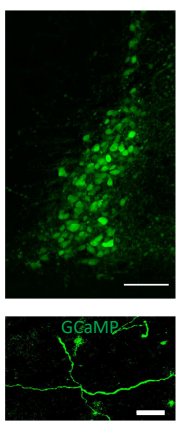
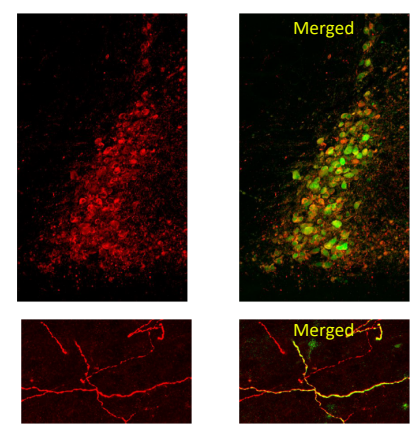

$\mathrm{F}$

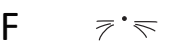

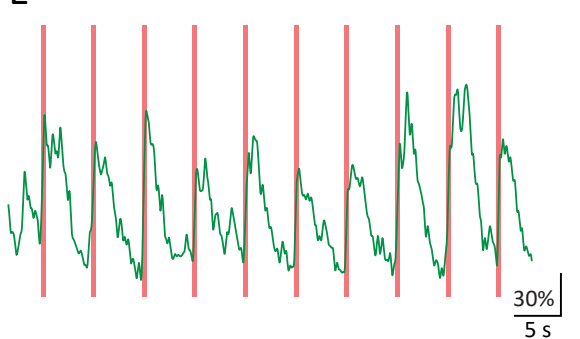

$\mathrm{H}$

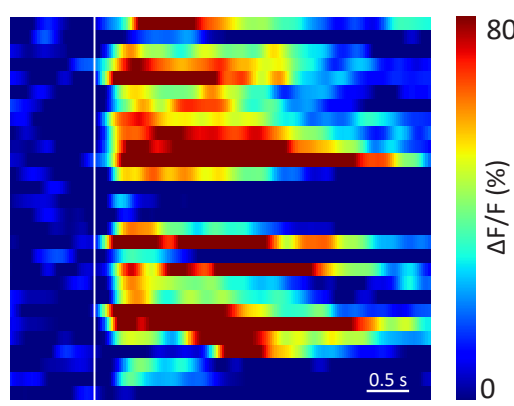

80

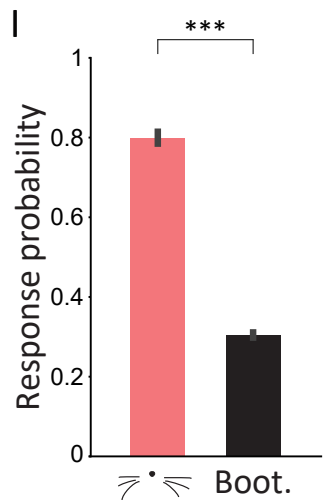

Figure 1. $\mathrm{Ca}^{2+}$ imaging of Locus Coeruleus axonal activity in the barrel cortex.

A. Noradrenergic (NE) axons in the barrel cortex labeled using a noradrenaline transporter (NET) antibody (red). Scale bar, $200 \mu \mathrm{m}$. B. Stereotactic injections of Flex-GCaMP6s into the LC of TH-IRES-Cre mice. C Top: viral expression of GCaMP6s in the LC (green), tyrosine hydroxylase (TH) antibody staining (red), and a merged image (yellow). Scale bar, $100 \mu \mathrm{m}$. Bottom: NE axons in the barrel cortex expressing GCaMP6s (green), NET antibody staining (red) and a merged image (yellow). Scale bar, $20 \mu \mathrm{m}$. D. Schematic illustration of two-photon $\mathrm{Ca}^{2+}$ imaging configuration from head-restrained mice, walking on a wheel. Inset: average image showing LC axons expressing GCaMP6s. E. An example fluorescence trace of an LC axon in response to whisker stimulation. Red lines indicate the period of whisker stimulation. F. Mean $\mathrm{Ca}^{2+}$ response of the same LC axon to whisker stimulation (S.E.M in green shaded area) G. Comparison of the mean evoked response to whisker stimulation and the bootstrap results for all LC axons. $\mathbf{H}$. Color raster plot of $\mathrm{Ca}^{2+}$ signals from all trials from the same axon, each row represents one trial. White 
line indicates the onset of the whisker stimulation. I. Comparison of response probability to whisker stimulation and bootstrap results for all LC axons.

\section{Response of LC axons to multiple stimuli in the barrel and visual cortices}

Primary sensory areas are notoriously known for their neurons highly selective responses (Kerr et al. 2007; Niell and Stryker 2008). In contrast, anatomically it has been shown that LC neurons receive inputs from many sources (Schwarz et al. 2015), and indeed electrophysiological recordings from the LC found neurons which respond to stimuli of two or more sensory modalities (Aston-Jones and Bloom 1981a). We have found that LC axons in barrel cortex reliably respond to somatosensory stimulation. The question is whether axons of LC neurons projecting to primary sensory areas show selectivity to that modality or do they respond to stimuli of other modalities as 145 suggested from the multimodal properties of LC neurons.

We therefore tested the responses to sensory stimuli from other modalities of LC axons in barrel cortex. We found that the same NE axons also responded to simple auditory and visual stimuli (see Methods) with a similar increase in $\mathrm{Ca}^{2+}$ signal (Figure 2A-B, Figure S2A-B). Given the recordings were made in a primary sensory area we were

150 surprised by the robustness and reliability of these cross-modal responses. These results suggest that NE may act as a multimodal signal, conveying information to primary sensory cortices about other modalities.

However, despite the robustness, we also noticed variations in the responses. When comparing the response of LC axons in barrel cortex to the different stimuli, we found 155 that the largest response was to the whisker stimulation, then to the auditory stimulation, and the weakest response was induced by the visual stimulation (Figure 2C, whisker: $26.16 \% \pm 1.38 \%, \mathrm{n}=78 \mathrm{ROIs}, 1,777$ trials, $15 \mathrm{FoV}, 4$ mice; auditory: $19.61 \% \pm 0.89 \%, n=92$ ROls, 2,707 trials, 15 FoV, 4 mice; visual: $10.02 \% \pm 1.57 \%$, $\mathrm{n}=53$ ROls, 1,031 trials, 8 FoV, 3 mice; $\mathrm{p}<0.001$; one-way ANOVA, Tukey's multiple

160 comparison test). Similar results were found when comparing the response probability of the different conditions (Figure S2C, whisker: $0.8 \pm 0.01, \mathrm{n}=78 \mathrm{ROIs}, 1,777$ trials, 15 FoV, 4 mice; auditory: $0.71 \pm 0.02, n=92$ ROls, 2,707 trials, 15 FoV, 4 mice; visual: $0.42 \pm 0.04, n=53$ ROls, 1,031 trials, 8 FoV, 3 mice; $p<0.001$, one-way ANOVA, Tukey's multiple comparison test).

Is this multimodal response specific to the barrel cortex or is this phenomenon similar across sensory cortices? To directly address this question, we imaged NE axons in the 
visual cortex and presented the mice with an identical set of sensory stimuli. Similar to the barrel cortex, in the visual cortex as well we found multimodal responses to whisker, auditory, and visual stimuli (Figure 2D, whisker: $31.22 \% \pm 2.27 \%, n=34$ ROIs, 573 trials, 7 FoV, 2 mice; auditory: $18.99 \% \pm 1.38 \%, n=44$ ROls, 709 trials, 10 FoV, 2 mice; visual: $4.21 \% \pm 0.7 \%, n=24$ ROls, 367 trials, 6 FoV, 2 mice; $p<0.001$, oneway ANOVA, Tukey's multiple comparison test). Similar results were also found when comparing the response probability of the different conditions (Figure S2D, whisker: $0.83 \pm 0.02, \mathrm{n}=34 \mathrm{ROIs}, 573$ trials, 7 FoV, 2 mice; auditory: $0.64 \pm 0.03, \mathrm{n}=44 \mathrm{ROIs}$, 175709 trials, 10 FoV, 2 mice; visual: $0.33 \pm 0.04, n=24$ ROIs, 367 trials, 6 FoV, 2 mice; $p<0.001$, one-way ANOVA, Tukey's multiple comparison test).

Remarkably, the LC axons in visual cortex responded in a similar manner to those in the barrel cortex, namely, the weakest response was to the visual stimulation, a larger response to the auditory stimulation and the largest response was to whisker

180 stimulation. These results may suggest that the variations in the axonal responses reflects the salience of the stimulation, independent of the modality of the primary sensory cortex where the axons project.

To test if the response of LC axons to a series of stimuli shows interesting dynamics (adaptation or facilitation), we compared the initial responses (the mean response to the first five trials with sensory stimulus) to the late responses (the mean response to the last five trials). Almost no change appeared in all three modalities (Figure 2E). As startle responses are highly adaptive (Ding et al. 2013) our results indicate that the NE axonal activity most likely does not represent a startle response.

As shown above, we found variations in the NE axonal responses to stimuli from 190 different modalities. We next examined if there are variations also in response to the same stimulus, depending on the behavioral state, namely, when the animal is running or standing. We therefore analyzed the response of LC axons in the barrel cortex to whisker stimulation while the mouse was running or standing. Interestingly, when whisker stimulation was presented during epochs where the mouse was running, there 195 was a very small axonal response compared to the response when the stimulus was presented while the mouse was stationary (Figure $2 \mathrm{~F}-\mathrm{H}$, whisker $\mathrm{r}_{\text {stat: }} 26.16 \% \pm 1.38 \%$, $\mathrm{n}=78$ ROls, 1,777 trials, 15 FoV, 4 mice; whisker $_{\text {run: }} 3.1 \% \pm 2.88 \%, n=20$ ROls, 32 trials, 5 FoV, 3 mice; $p<0.001$, t test). Similar results were found for auditory and

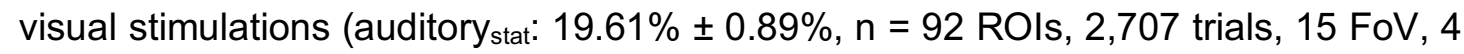
200 mice, auditory run: $-6.07 \% \pm 1.63 \%, \mathrm{n}=26$ ROls, 65 trials, 6 FoV, 3 mice, $\mathrm{p}<0.001, \mathrm{t}$ test; visual ${ }_{\text {stat: }} 10.02 \% \pm 1.57 \%, \mathrm{n}=53 \mathrm{ROIs}, 1,031$ trials, $8 \mathrm{FoV}, 3$ mice, visual ${ }_{\text {run }}$ : 
$3.53 \% \pm 1.76 \%, n=35$ ROls, 69 trials, 5 FoV, 2 mice; $p<0.01$, $t$ test). As previously reported (Reimer et al. 2016), at the beginning of walking the activity of NE axons increases resulting in a relative large shift in baseline activity (Figure $2 \mathrm{G}$ ). Thus, the decrease in response during running (Figure $2 \mathrm{H}$ ) may result from a saturation in the $\mathrm{Ca}^{2+}$ sensor itself (ceiling effect) or alternatively reflect a more general phenomenon where sensory stimulation during active states results in weaker responses (Petersen et al. 2003).

A

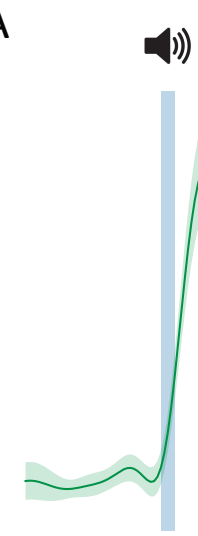

C
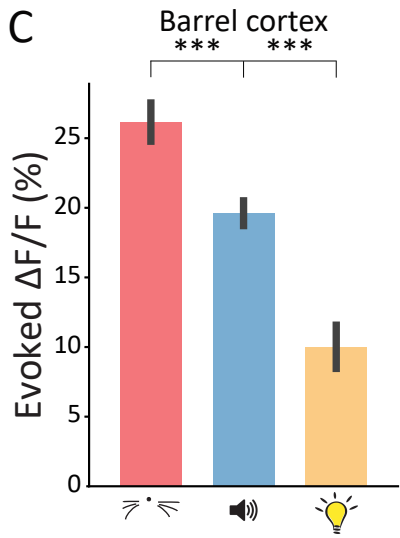

$\mathrm{F}$

$\mathrm{F} \Delta \mathrm{F} / \mathrm{F}$

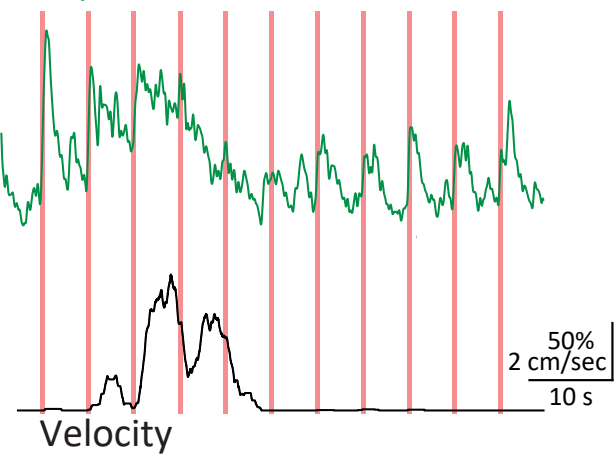

B
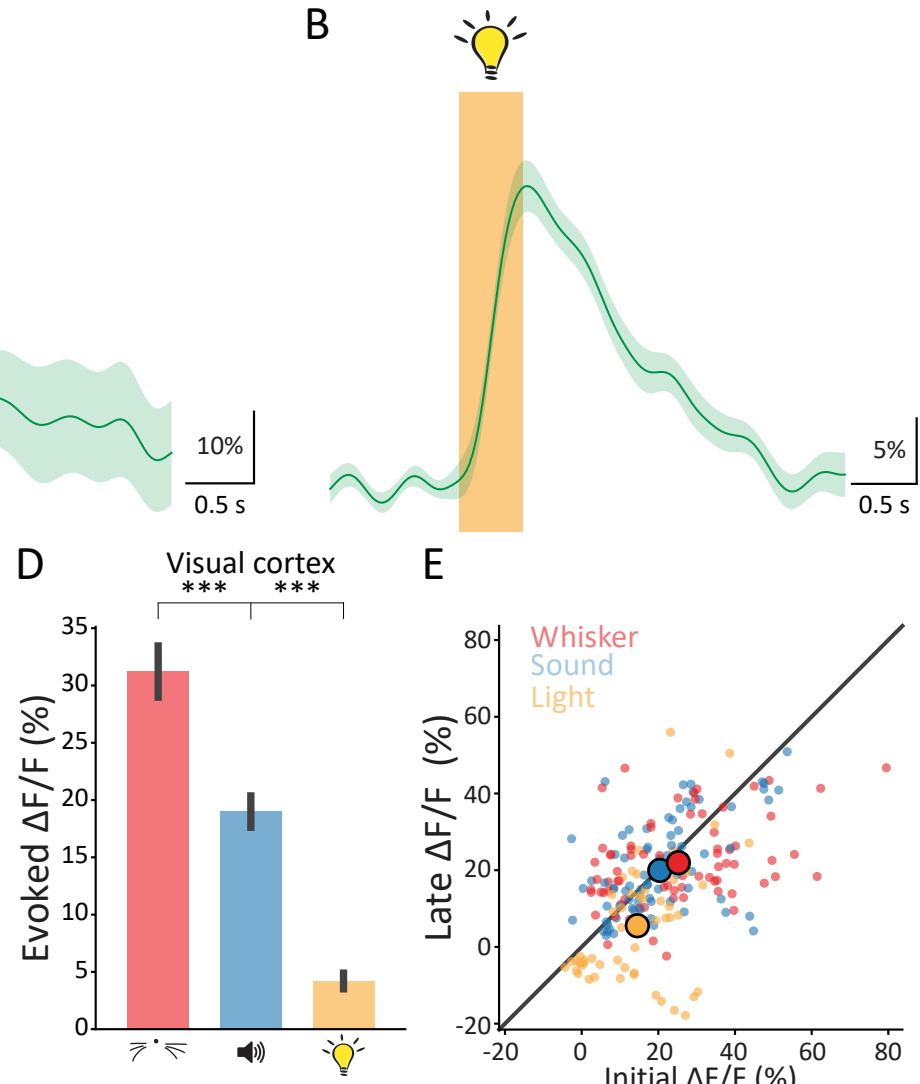

$\mathrm{E}$

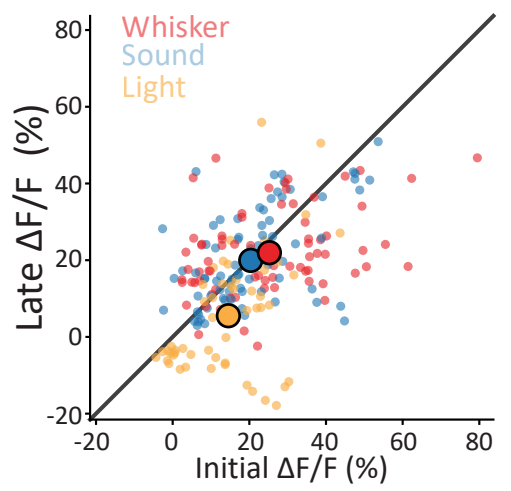

$\mathrm{H}$

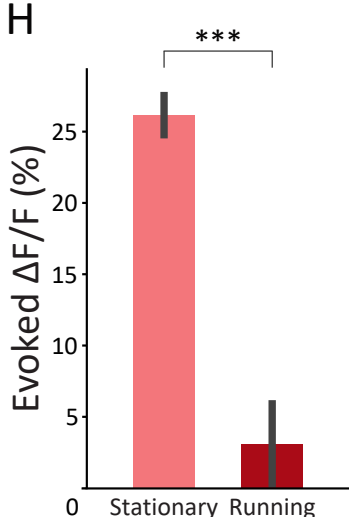

Figure 2. Response of LC axons to multiple stimuli modalities in the barrel and visual cortices. A. Mean $\mathrm{Ca}^{2+}$ response (solid line) of the same LC axon from Figure 1 (barrel cortex) to auditory stimulation. Blue shaded area indicates the time and 
duration of the auditory stimulation (SEM in shaded green) B. Mean $\mathrm{Ca}^{2+}$ response of the same axon to visual stimulation. Yellow shaded area indicates the time and duration of the visual stimulation. C. Comparison of the mean evoked response between the three conditions (whisker, auditory and visual stimulations) in the barrel cortex D. Comparison of the mean evoked response between the three conditions (whisker, auditory and visual stimulations) in the visual cortex. E. Comparison of the mean response of the five initial responses of $L C$ axons and the mean response of the five latest responses. F. Top, $\mathrm{Ca}^{2+}$ fluorescence traces of an $\mathrm{LC}$ axon in response to whisker stimulation (green). Bottom, velocity of the mouse (black). Red shaded areas indicate whisker stimulations. G. Comparison of the baseline activity in LC axons in the barrel cortex during stationary and running epochs. $\mathbf{H}$. Comparison of the mean evoked response in LC axons in the barrel cortex during stationary and running epochs.

\section{Experience dependent plasticity in NE axons activity following associative fear}

Our data demonstrate that the LC axonal activity in sensory cortices conveys a robust multimodal spatially-diverse signal. The data presented in figure 2 also hint that some information is carried at a finer scale of variation. First, there are consistent variations in the responses to stimuli of different modalities (Figure 2C, D). Second, the responses to the same stimulus change between behavioral states (Figure $2 F, H$ ). Are the responses of LC axons capable of showing experience dependent plasticity such that they can deliver information on sensory cues at this finer resolution (beyond the mere appearance of a salient sensory stimulus)?

To directly address this question, we trained mice in an associative auditory fear 220 conditioning (FC) paradigm and measured the freezing behavior of mice in response to the conditioned stimulus (CS+) as compared to a non-associated stimulus (CS-; not paired with a foot shock, Figure $3 \mathrm{~A}$ ). Notably, under freely behaving conditions, the mice showed a prominent and discriminative fear response to the CS+ (Figure 3B, CSfreezing: $20.86 \% \pm 2.97 \%, \mathrm{CS}^{+}{ }_{\text {freezing }}: 71.75 \pm 5.11 \%, n=21$ mice; $p<0.001$, paired $t$ test),

225 indicating successful fear memory learning. To estimate fear expression in head-fixed mice, we measured the change in pupil diameter in response to the auditory cues (CS ${ }^{-}$ versus $\mathrm{CS}^{+}$) during habituation and recall (Abs et al. 2018; Garcia-Junco-Clemente et al. 2019, Figure 3C). Following fear conditioning, the pupil evoked responses increased for the $\mathrm{CS}^{+}$but not for the $\mathrm{CS}^{-}$(Figure 3D-F; $\mathrm{CS}^{-}$before: $0.46 \% \pm 1.46 \%$, $230 \mathrm{CS}^{+}{ }_{\text {before: }}-0.57 \% \pm 0.62 \% ; n=7$ mice, 263 trials; $p=0.47$, paired t test; CS $^{-}{ }_{\text {after }}: 1.88 \%$ 
$\pm 0.45 \%, \mathrm{CS}^{+}$after: $10.2 \% \pm 2.15 \%, n=9$ mice, 352 trials; $p<0.01$ paired $t$ test). Thus, pupil response can be used as a faithful readout of fear conditioning.

To test whether NE axons in the barrel cortex show a discriminative response following auditory fear conditioning we combined the fear conditioning behavior with in vivo twophoton $\mathrm{Ca}^{2+}$ imaging of $\mathrm{LC}$ axons (Figure $3 \mathrm{G}$ ). During fear memory recall, NE axons showed a larger $\mathrm{Ca}^{2+}$ increase for the $\mathrm{CS}^{+}$than for the $\mathrm{CS}^{-}$(Figure $3 \mathrm{H}-\mathrm{I}, \mathrm{CS}^{-}: 5.16 \% \pm$ $1.64 \%, \mathrm{CS}^{+}: 9.24 \% \pm 1.6 \%, \mathrm{n}=18 \mathrm{ROIs}, 168$ trials, 11 FoV, 3 mice; $\mathrm{p}<0.01$ paired $\mathrm{t}$ test). Consistent with this result, mice that experienced fear conditioning but failed to form a stable memory, showed no significant difference in $\mathrm{Ca}^{2+}$ response following both 240 CSs (Figure S3A, CS: $2.31 \% \pm 0.92 \%, \mathrm{CS}^{+}: 2.61 \% \pm 1.04 \%, \mathrm{n}=22 \mathrm{ROIs}, 211$ trials, 8 FoV, 2 mice; $p=0.72$ paired t test), further supporting the interpretation that the potentiation in LC axonal responses resulted from the association of the CS+ auditory stimulus with fear. These data demonstrate that recall of an aversive auditory memory is associated with a selective pronounced $\mathrm{Ca}^{2+}$ increase in NE axons located in the 245 cortex. More generally, these results show that the robust multimodal spatially-diverse signal of the cortical projections of the NE system, is plastic and can modify the information it conveys in a behaviorally meaningful manner. 
A

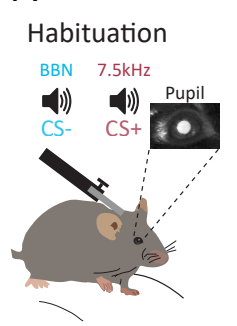

C

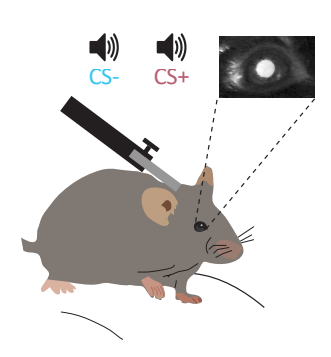

Conditioning

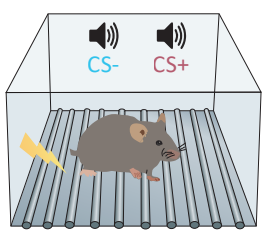

Recall head-fixed Recall freely behaving
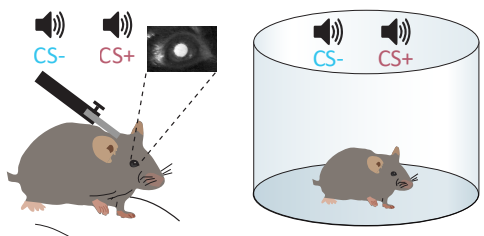

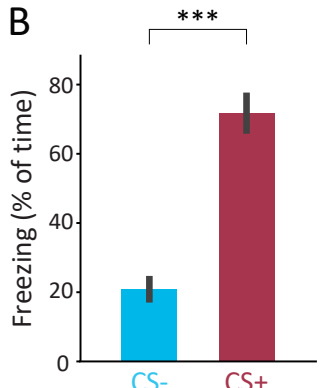

After FC

$E$

D Before FC

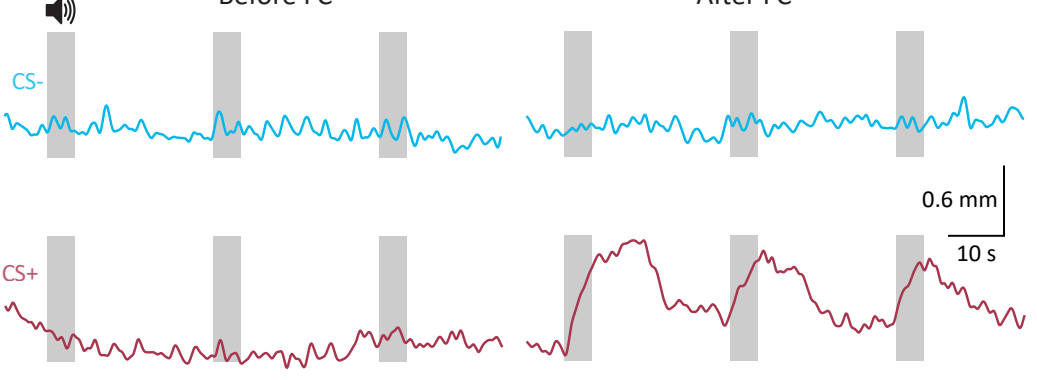

$\mathrm{F}$ After FC

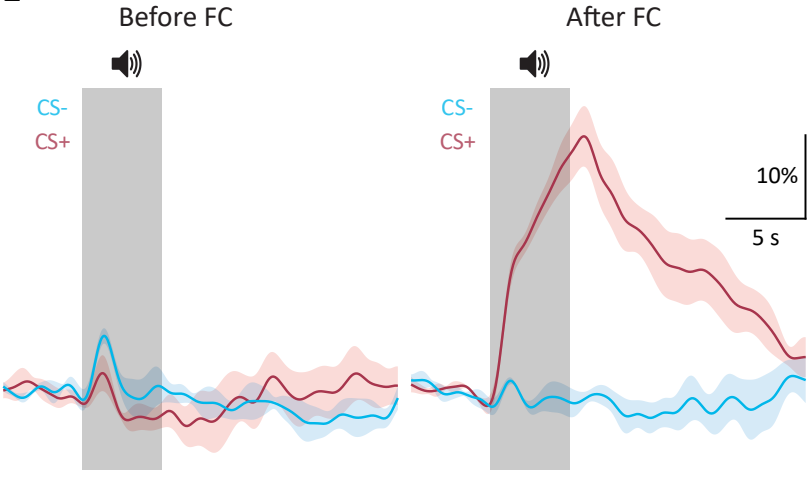

G

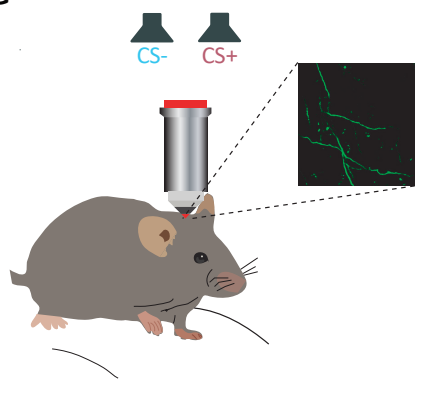

$\mathrm{H}$

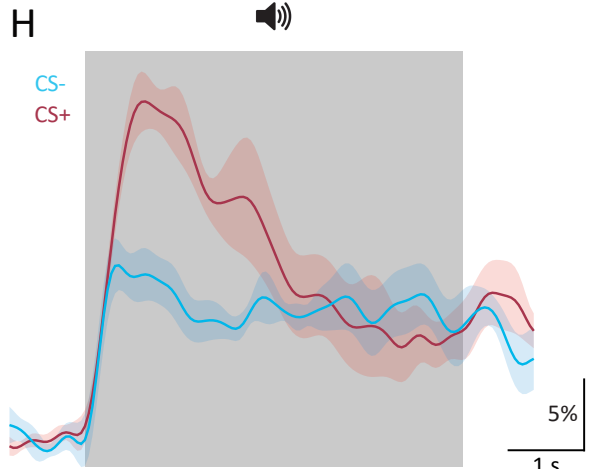

Before FC
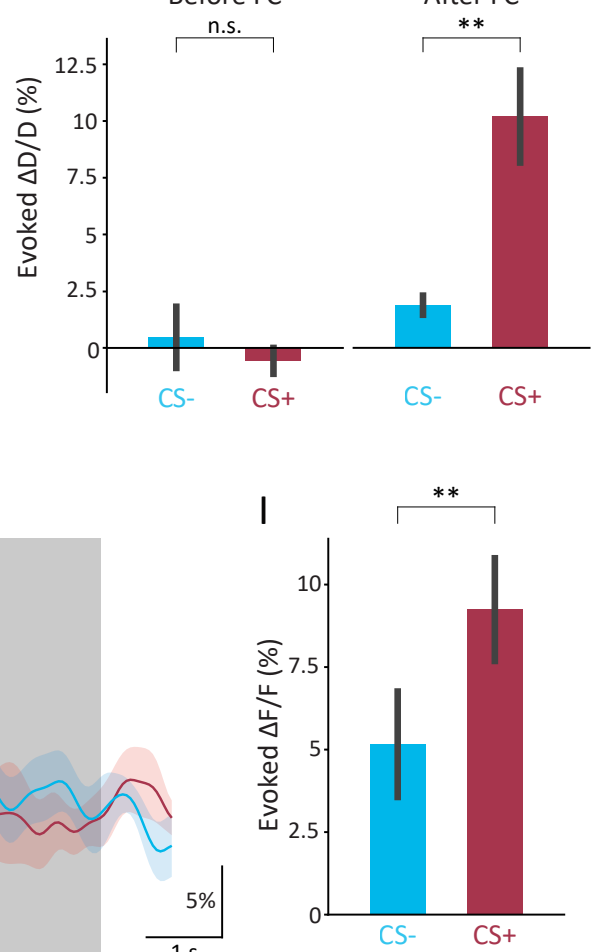

Figure 3. Experience dependent plasticity in LC-axon activity following associative fear acquisition. A. Schematic illustration of the associative auditory fear conditioning paradigm. B. Comparison of the freezing behavior (in a freely behaving memory recall session) to the conditioned stimulus (CS+) and to a nonconditioned stimulus (CS-; not paired with a foot shock). C. Schematic illustration of a memory recall session in a head-fixed mouse. D. Examples of pupil diameter responses to $\mathrm{CS}^{-}$(blue) and $\mathrm{CS}^{+}$(red) before (left) and after (right) fear conditioning 
(FC). Gray shaded area indicates the CSs presentations. E. Mean pupil response to the CSs before (left) and after (right) FC in one mouse. Red- and blue-shaded areas are the S.E.M. F. Comparison of the mean evoked pupil response before (left) and after (right) FC in all mice. G. Schematic illustration of two-photon $\mathrm{Ca}^{2+}$ imaging in head-restrained mice during memory recall. $\mathbf{H}$. Mean $\mathrm{Ca}^{2+}$ response of $\mathrm{LC}$ axons to CSs during memory recall in one mouse. I. Comparison of the mean evoked $\mathrm{Ca}^{2+}$ response to the CSs in all mice.

\section{Nonlinear relationship between auditory evoked LC axonal and local dendritic responses in barrel cortex}

Having established evoked responses of NE axons activity in the barrel cortex, we next aimed to explore the relationship between noradrenergic axon transient responses and the local circuit activity. Previously it has been suggested that the excitability of dendrites located in the upper layers of the cortex is subject to neuromodulation (Barth et al. 2008; Dembrow et al. 2010; Labarrera et al. 2018; Williams and Fletcher 2019). We thus expressed the red-shifted genetically encoded calcium indicator, jRGECO1a (Dana et al. 2016), in neurons located in the barrel cortex and GCaMP6s in the LC (Figure 4A-B). This allowed us to perform dual-color functional imaging, of both LC axons and dendritic branches of neurons in layer 1 of the barrel cortex. Imaging of spontaneous activity showed that $\mathrm{Ca}^{2+}$ events in $\mathrm{NE}$ axons were correlated with $\mathrm{Ca}^{2+}$ events in the local dendrites (Figure 4C). The cross-correlation function between LC axons activity and the mean dendritic activity, showed a large peak close to zero lag (Figure 4D, 15 axonal ROls, 52 dendritic ROls, $5 \mathrm{FoV}, 3$ mice). While this large peak at this lag indicates a strong temporal correlation between the two signals, due to the different rise and decay time-constants of jRGECO1a (Dana 2016) and GCaMP6s (Chen et al. 2013), we were unable to determine the precise temporal relationship between the NE axonal and local dendritic activity.

As we have shown above, LC axons in the barrel cortex also respond to auditory stimulation (Figure 2). Thus, we tested the response of local dendritic branches to auditory stimulation and found that a subset of these dendrites indeed responded to auditory stimuli as well. However, the response reliability of these dendrites was not very high and they did not respond in all trials of stimulation (Figure 4Es). To check if variations in NE activity could explain some of this unreliability, we compared the response probability of a dendrite when the axonal response was weak ( $1^{\text {st }}$ quartile;

275 see Methods) and when the axonal response was strong ( $4^{\text {th }}$ quartile). Notably, the dendritic response probability was larger when the axonal response was strong (Figure 
4F, weak response: $0.39 \pm 0.04$, strong response: $0.81 \pm 0.04, n=26$ dendritic ROIs, 27 axonal ROls, 356 trials, 9 FoV, 3 mice; $p<0.001$, paired t test; Figure S4A). Moreover, the mean evoked dendritic response was also larger when axonal response was large (Figure S4B, weak response, $4.54 \% \pm 0.8 \%$, strong response, $11.56 \% \pm$ $1.28 \%, \mathrm{n}=26$ dendritic ROls, 27 axonal ROls, 356 trials, 9 FoV, 3 mice; $p<0.001$, paired $t$ test; See also Figure S4C for quantification of the wide dynamic range of the axonal fluorescent signal).

Finally, we examined the response probability of these barrel cortex dendrites as a function of the NE axonal response (Figure 4G). Strikingly, as the axonal response increased so did the probability of the dendrites to respond (to auditory stimuli). This relationship is not linear, showing saturation at both low and high ends and was fitted with a sigmoidal function. These data indicate that dendritic response probability in the barrel cortex to an auditory stimulation, increases when NE activity is higher, suggesting that the transient variations in NE concentration in the cortex following a sensory stimulation may explain part of the dendritic responses.

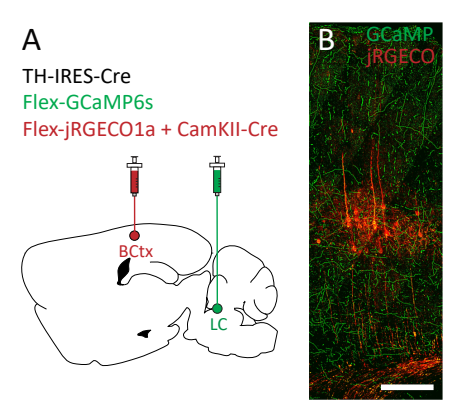

E

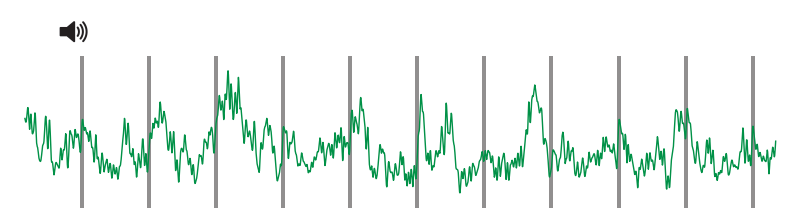

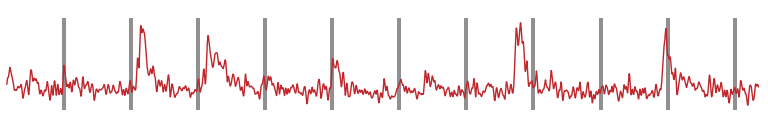

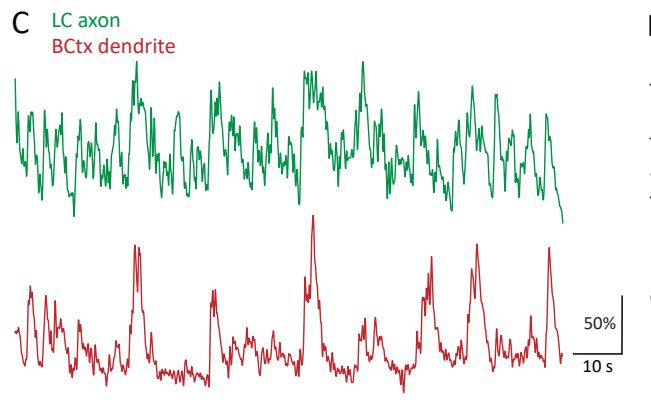

$\mathrm{F}$

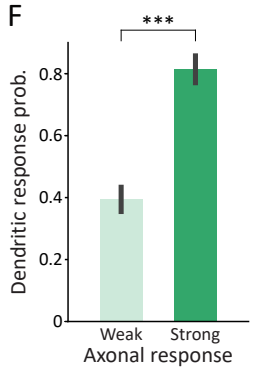

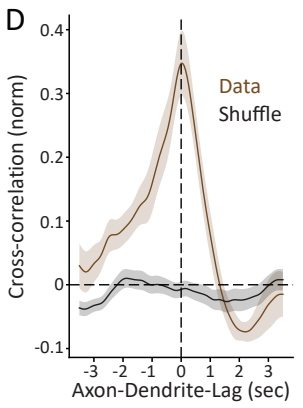

G

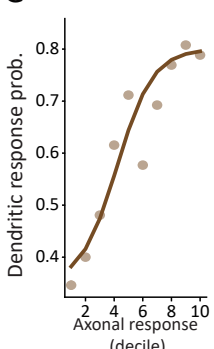

Figure 4. Relationship between the sensory responses of LC axons and local barrel cortex dendrites. A. Stereotactic injections of Flex-jRGECO1a mixed with CamKII-Cre into the barrel cortex and Flex-GCaMP6s into the LC of TH-IRES-Cre mice. B. Noradrenergic axons in the barrel cortex expressing GCaMP6s (green) and neurons in the barrel cortex expressing jRGECO1a (red). Scale bar, $200 \mu \mathrm{m}$. C. 
Dual-color two-photon imaging of $\mathrm{NE}$ axons and local dendrites. Example $\mathrm{Ca}^{2+}$ fluorescence traces of an LC axon (green) and of a local dendrite (red). D. Mean cross-correlation between the mean activity of LC axons and the mean activity of an adjacent dendrites (brown). Same analysis for shuffled data in black. Shaded areas are the S.E.M. E. Example $\mathrm{Ca}^{2+}$ fluorescence traces of an LC axon (green) and of a local dendrite (red) in response to auditory stimulation. Gray shaded area indicates the period of the auditory stimulation. F. Comparison of the mean dendritic response probability when axonal response is weak and strong. G. The mean dendritic response probability as a function of the axonal response. Dark brown line is a sigmoid fit.

\section{Discussion}

295 In this study, we examined the activity of noradrenergic LC axons in primary sensory cortices. We imaged the $\mathrm{Ca}^{2+}$ activity of $\mathrm{NE}$ axons in awake mice and found a multimodal response to somatosensory, auditory and visual stimulations in the barrel cortex. Similar results were observed also in the visual cortex. These results suggest that NE acts as a multimodal spatially diverse signal, conveying integrated information

300 from distinct sensory modalities to different areas of the sensory cortex. Moreover, the auditory response can be modulated following an associative auditory fear conditioning experience. Namely, NE axons show a selective $\mathrm{Ca}^{2+}$ increase for a conditioned stimulus $\left(\mathrm{CS}^{+}\right)$after it was paired with a foot shock. Finally, we simultaneously investigated the responses of LC axons and local dendrites in the barrel cortex to 305 auditory stimulation and found that the response probability of barrel cortex dendrites to auditory stimulation, increases monotonically and nonlinearly with the $\mathrm{Ca}^{2+}$ activity of noradrenergic LC axons.

\section{NE axons show multimodal spatially-diverse reliable response}

The noradrenergic system is multimodal in various respects. Electrophysiological recordings in the LC of rats have shown responses to simple stimuli of different modalities (Aston-Jones and Bloom 1981a). In addition, anatomical studies (Kim et al. 2016) have shown that individual LC neurons may project to more than one primary sensory area. Thus, LC neurons receive input from and respond to various modalities and project to various targets where sensory information is processed. Our results are consistent with these findings, and show functionally that LC axons in primary sensory cortices respond to sensory stimuli of various modalities. This may suggest that NE activity is a nonspecific broad signal that conveys little information. However, the 
details of the responses of NE signals as we observe, show interesting variations to behavioral states (Figure $2 \mathrm{H}$ ), to the modality or saliency of the stimulus (Figure $2 \mathrm{C}$ ) and also plasticity that corresponds to behavioral importance (Figure 3I). Moreover, the variation in the NE signal predicts in a nonobvious way the activity of neurons in the local circuit (Figure 4G). Therefore, these data suggest that understanding the contribution of the NE signals to cortical processing requires high resolution sensitivity to small nuances in the NE signal variations.

\section{Relationship between spontaneous NE activity and cortical circuit activity.}

Most of what is known about the relationship between NE and activity of local cortical circuits relies on using pupil diameter measurements as a proxy to NE activity (AstonJones and Cohen 2005; Varazzani et al. 2015; Joshi et al. 2016; Reimer et al. 2016). Simultaneous recordings of neuronal activity in auditory cortex with pupil diameter, shows that brief pupil microdilations were associated with $5-20 \mathrm{mV}$ depolarization in the membrane potential of cortical neuronal (McGinley et al. 2015). In both visual and somatosensory cortices, small spontaneous fluctuations in pupil diameter track changes in the intracellular dynamics of L2/3 neurons (Reimer et al. 2014). Our results on the high correlation between spontaneous NE axons activity and local dendritic activity is consistent with these results.

\section{NE signals and dendritic computation in perceptual sensitivity}

NE is crucial for maintaining cognitive brain functions such as perception, attention, and learning (Berridge and Waterhouse 2003; Aston-Jones and Cohen 2005; Sara 2009; Sara and Bouret 2012). A recent study demonstrated that optogenetic 340 stimulation of LC neurons, improves perceptual sensitivity in rats (Rodenkirch et al. 2019) and other reports showed that LC activation accelerates perceptual learning in the auditory system (Martins and Froemke 2015; Glennon et al. 2019). Moreover, there is evidence for noradrenergic involvement in perceptual shifts in human subjects (Einhäuser et al. 2008). However, there is little mechanistic understanding of how NE 345 changes contribute to these effects on perception at the circuit and cellular level. One candidate mechanism is the contribution of NE to synergistic integration of information from different sources through its effect on dendritic excitability (Barth et al. 2008; Phillips et al. 2016, 2018; Labarrera et al. 2018).

Several studies in awake mice indicate that $\mathrm{Ca}^{2+}$ activity in the dendrites of cortical

pyramidal cells is elevated during cognitive tasks, and that manipulating the activity of these dendrites shifted the perceptual threshold (Xu et al. 2012; Cichon and Gan 2015; Manita et al. 2015; Miyamoto et al. 2016; Takahashi et al. 2016; Ranganathan et al. 
2018). Here, we show that the probability of a dendritic response to auditory stimulation increases monotonically with the activity levels of NE axons, suggesting that NE can

355 act as a signal that lowers the threshold for the generation of dendritic $\mathrm{Ca}^{2+}$ events. Our study is in line with the proposal that large $\mathrm{Ca}^{2+}$ events in the dendrites are tuned by NE and that the sensitivity of the dendrites is modulated by NE (Phillips et al. 2016, 2018; Labarrera et al. 2018). Future experiments aimed at establishing this hypothesis will ideally include recordings from both NE signals and local dendritic activity in awake, behaving animals while they engage in a perceptual task. 


\section{Supplementary figures}
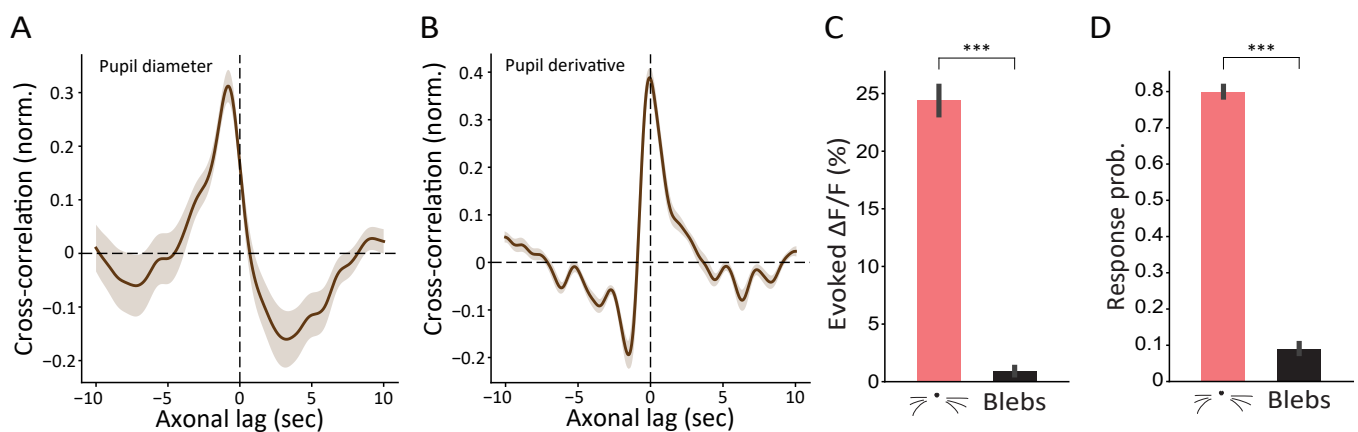

Supplementary Figure 1. Correlation between pupil diameter and NE axons. Comparison of NE axons response and blebs response to whisker stimulation in the barrel cortex. A. Cross-correlation between pupil diameter and NE axons. B. Cross-correlation between pupil diameter derivative and NE axons. C. Comparison of the mean evoked response of NE axons and blebs to whisker stimulation. $\mathbf{D}$. Comparison of the response probability of NE axons and blebs to whisker stimulation. 

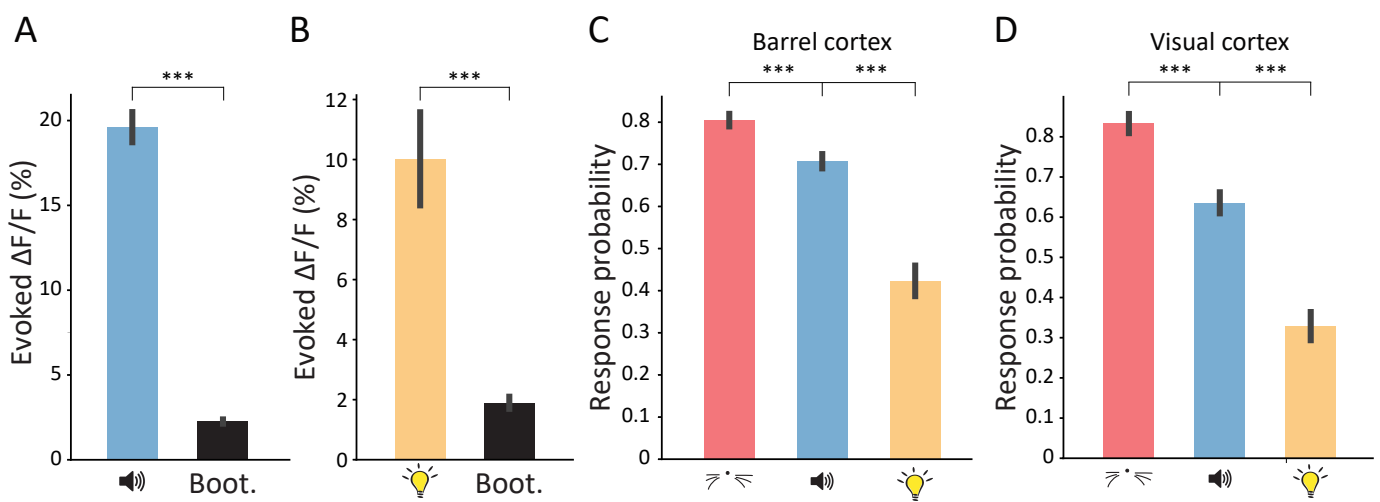

Supplementary Figure 2. Response of LC axons to multiple stimuli in the barrel and visual cortex. A. Comparison of the mean evoked response to auditory stimulation and the bootstrap results for all LC axons. B. Comparison of the mean evoked response to visual stimulation and the bootstrap results for all LC axons. C. Comparison of the response probability between the three conditions (whisker, auditory and visual stimulations) in the barrel cortex. D. Same as $\mathrm{C}$ but in the visual cortex. 

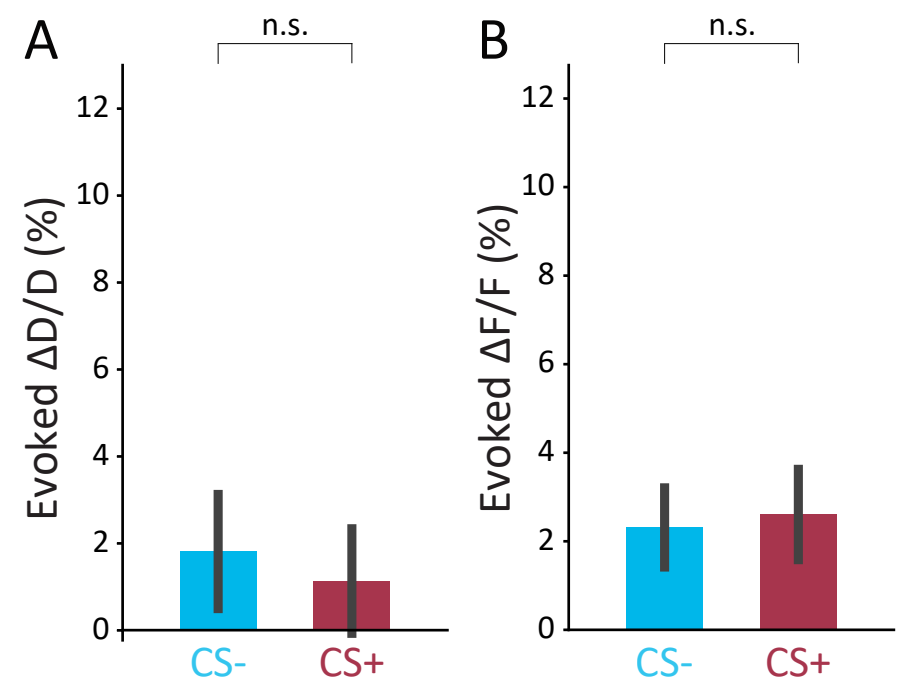

Supplementary Figure 3. Pupil and NE axonal responses to auditory cue in mice that experienced fear conditioning but failed to form a stable memory. A Comparison of the mean evoked pupil response in mice that failed to form a stable memory. B. Comparison of the mean evoked $\mathrm{Ca}^{2+}$ response in mice that failed to form a stable memory. 
A

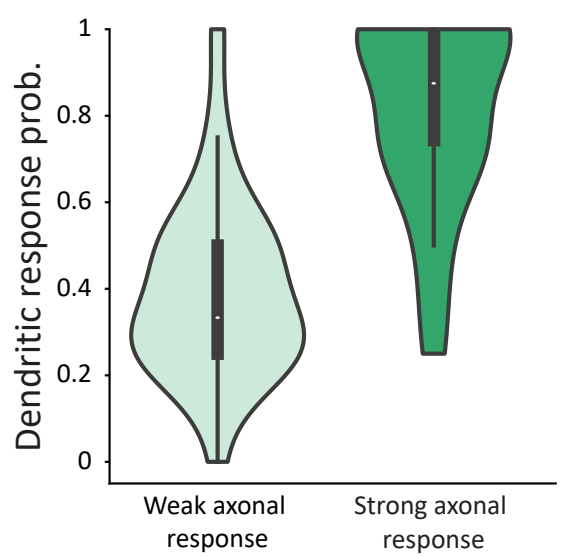

B

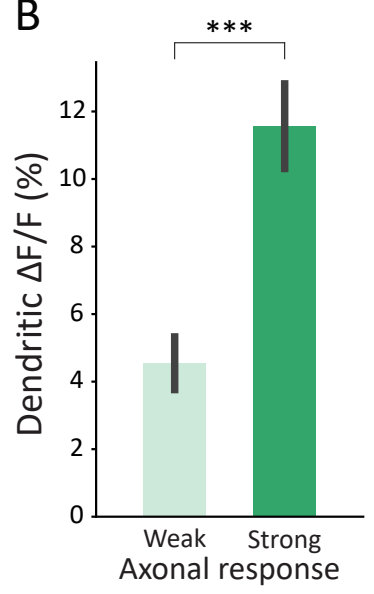

C

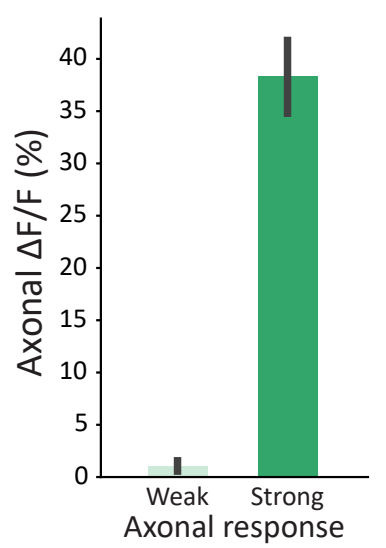

Supplementary Figure 4. Comparison of dendritic response probability during weak and strong axonal responses. A. Violin plot showing the distribution of dendritic response probability when axonal response is weak or strong. The small black box shows the quartiles of the dataset and the whiskers extend to show the rest of the distribution. B. Comparison of the mean dendritic evoked response when axonal response is weak and strong. C. Comparison of the mean axonal evoked response when axonal response is weak $(1.06 \% \pm 0.5 \%)$ or strong $(38.28 \% \pm$ $3.55 \%$ ). 


\section{Acknowledgments}

Inbal, Michael (pupil software), Tali kimchi

\section{Methods}

\section{Animals}

We used TH-IRES-Cre mice (Lindeberg et al. 2004), 8-13 weeks old. The Hebrew University Animal Care and Use Committee approved all experiments.

\section{Surgery and Viral Vector Injections}

Viral vectors were delivered using standard stereotactic injections to the right locus coeruleus (Carter 2010, anteroposterior: $-5.45 \mathrm{~mm}$; mediolateral: $1.28 \mathrm{~mm}$; dorsoventral: $3.65 \mathrm{~mm}$ ) under isoflurane anesthesia. Expression of GCaMP6s (Chen et al., 2013) in LC neurons was achieved via viral injection of pAAV.CAG.Flex.GCaMP6s.WPRE.SV40 (AAV9) (Addgene). For sparse labeling of neurons in the barrel cortex with jRGECO1a (Dana 2016), pAAV.CAG.Flex.NESjRGECO1a.WPRE.SV40 (AAV1; Addgene) was co-injected with diluted (1:10) AAV9.CamKII.Cre. A head-post was installed after viral injection.

Four weeks after the virus injection, a 3-mm craniotomy was drilled and a cranial 385 window consisting of two stacked 3-mm coverslips under a 5-mm coverslip were sealed into the craniotomy.

\section{Two-Photon Calcium Imaging}

Imaging from awake animals was performed with a low-power temporal oversampling 390 (LOTOS) two-photon microscope (LotosScan2015, Suzhou Institute of Biomedical Engineering and Technology; http://english.sibet.cas.cn/) at $920 \mathrm{~nm}$ with a Ti:Sapphire laser (Vision II, Coherent, CA) and imaged through a 25x, 1.05 numerical aperture (NA) water immersion objective (Olympus, Japan). Frame images were acquired at 20-40 frames per second.

395 For dual-color imaging (Figure 4) a single excitation source at $1000 \mathrm{~nm}$ was used to excite both indicators. 


\section{Fear conditioning}

After mice were habituated to head-fixation under the microscope, both CSs (CS+: pure tone, $7.5 \mathrm{kHz}$; CS-: broadband noise $(\mathrm{BBN}))$ were presented for habituation of the CSs. Each CS was presented 18-24 times (each presentation 5 sec long, 25 sec intertrial interval). CS+ and CS- were presented in an alternating fashion.

The Fear Conditioning (FC) apparatus consisted of a conditioning box $(18 \times 18 \times 30 \mathrm{~cm})$, with a grid floor wired to a shock generator surrounded by an acoustic chamber (Ugo 405 Basile), and controlled by the EthoVision software (Noldus). After head-fixed habituation, mice were placed in the conditioning box and CSs were presented at random times. For the CS+ a pure tone $(7.5 \mathrm{kHz})$ was presented for $20 \mathrm{sec}$, followed by a 2 sec foot shock (0.6 mA, repeated 3-5 times). For the CS- a broadband noise was presented for $20 \mathrm{sec}$ but was never associated with a foot shock (10-12 times). The inter-trial interval was random selected between 1-2 min.

To test $\mathrm{FC}$ recall, mice were placed in a different context (a cylinder-shaped cage with stripes on the walls and a smooth floor), and then presented with the CSs. Each CS was presented for $5 \mathrm{sec}$ and the inter-trial interval was $25 \mathrm{sec}$ (repeated 5 times). Conditioned freezing was measured during the $5 \mathrm{sec}$ presentation of the CS (and

415 averaged over trials). Freezing was automatically measured throughout the testing trial by the EthoVision tracking software. For head-fixation recall, the same CSs were presented (5 sec long, 25 sec inter-trial-interval, repeated 18-24 times) and freezing was measured by pupil diameter.

\section{Immunostaining}

For immunostaining of LC neurons and NE axons, $50-\mu \mathrm{m}$ thick coronal sections were collected sequentially into phosphate buffered saline (PBS). Then, sections were washed in PBS and blocked for 2-3 $\mathrm{h}$ at room temperature in 10\% normal donkey serum (NDS) in PBS with $0.3 \%$ Triton-X100 (PBST). For immunostaining of LC 425 neurons, primary antibodies for tyrosine hydroxylase were used (rabbit anti-tyrosine hydroxylase, Millipore, AB152, 1:2,000). For immunostaining of NE axons in the cortex, primary antibodies for noradrenaline transporter were used (mouse anti-noradrenaline transporter was used, PhosphoSolutions, 1447-NET, 1:10000; rabbit anti-GFP, novusbio NB600-308, 1:1,000). The primary antibodies were diluted in 5\% NDS in

430 PBST and incubated overnight at room temperature with mild shaking. After $3 \times 10$ min washes in PBST, secondary antibodies (donkey anti-rabbit, Alexa-488, 1:500 and 
donkey anti-mouse, Alexa-647, 1:500, Jackson ImmunoResearch) were applied for 1$2 \mathrm{~h}$ at room temperature with mild shaking, followed by 3X10 min washes in PBST. The sections were mounted and imaged with a confocal microscope (FV-10i 435 Olympus). Images were processed using ImageJ software.

\section{Head-fixed sensory stimuli}

Whiskers were stimulated by air puffs for a duration of 200-500 ms. Air puffs were delivered from a compressed air tank to a tube ending with a pipette tip facing the 440 mouse's whiskers. For auditory stimulation a broadband noise was presented with speakers for $100 \mathrm{~ms}$. Visual stimulation was delivered by presenting light flashes with a green LED for $200-500 \mathrm{~ms}$.

\section{Pupil}

445 Recording of pupil diameter was performed using a camera (ELP-USBFHD01M-L36120FPS) at $20 \mathrm{~Hz}$. A moderate level of ambient illumination was maintained by $410-$ $420 \mathrm{~nm}$ high power LEDs (743-IN-K2PUV-U70, Inolux, Mouser Electronics). Pupil segmentation was performed with SimplePupil - a LabVIEW-based pupil tracker for neuroscience applications developed in Ilan Lampl's Lab.

450 The evoked change in pupil diameter, $\Delta D / D_{0}$ (percent) was calculated as $\left(\left(D-D_{0}\right) / D_{0}\right)$ * 100 , where $D_{0}$ is the mean activity during the 2 sec preceding the stimulus. The mean evoked response was calculated as the mean $\Delta \mathrm{D} / \mathrm{D}_{0}$ during the $10 \mathrm{sec}$ following stimulus onset.

\section{Data Analysis}

\section{$\mathrm{Ca}^{2+}$ Imaging}

All analyses were performed using ImageJ software (Schneider et al., 2012) and custom-written codes in Python. Movements were corrected using the moco plugin (Dubbs et al., 2016). ROls were manually selected, and fluorescence traces were low pass-filtered at $3 \mathrm{~Hz}$. The evoked fluorescence change, $\Delta F / F_{0}$ (percent) was calculated as $\left(\left(F-F_{0}\right) / F_{0}\right) * 100$, where $F_{0}$ is the mean activity during the 1 sec preceding the stimulus. In spontaneous fluorescence traces, $F_{0}$ was defined as the tenth percentile of $F$. The mean evoked response was calculated as the mean $\Delta F / F_{0}$ during the 2 sec 
following stimulus onset. To avoid the possibility of visual stimulation affecting the

465 imaging fluorescence, in figure 2 the mean evoked response was calculated as the mean $\Delta \mathrm{F} / \mathrm{F}_{0}$ only starting $0.5 \mathrm{sec}$ following stimulus onset for all conditions. In figure $3 \mathrm{I}$ the mean evoked response was calculated as the mean $\Delta F / F_{0}$ during the entire auditory stimulation (5 sec). All stimulation occurring immediately before or during running periods were excluded from the analysis.

470 We defined a response to a stimulation if the mean evoked response was larger than $8 \%$ fluorescence (changing the threshold yielded similar results). The response probability was defined as the mean of responses over all trials. Bootstrap was done by shuffling the times of stimulations.

In figure $4 \mathrm{E}-\mathrm{F}$, dendrites that responded in more than $40 \%$ of the trails were selected

475 for analysis. In figure 4E, 'weak axonal response' was defined as the mean evoked response in trials were the axonal response was in the first quartile of responses, while the 'strong axonal response' was defined as the mean evoked response in trials were the axonal response was in the fourth quartile of responses. Each axon was discretized separately. In figure 4F axonal responses were discretized based on the deciles.

\section{Statistical Analysis}

Statistical significance was assessed using an unpaired Student's two-sided t test. In figure $3 B, F, I$, and figure $4 E$ a paired $t$ test was used. In figure $2 C-D$, $p$ values were computed using analysis of variance (ANOVA) with Tukey post-hoc test. Significance 485 level were marked as ${ }^{*} p<0.05,{ }^{* *} p<0.01$, and ${ }^{* * *} p<0.001$. All data are reported as mean \pm S.E.M unless otherwise specified. 


\section{References}

490

Abs E, Poorthuis RB, Apelblat D, Muhammad K, Pardi MB, Enke L, Kushinsky D, Pu D-L, Eizinger MF, Conzelmann K-K, Spiegel I, Letzkus JJ. 2018. LearningRelated Plasticity in Dendrite-Targeting Layer 1 Interneurons. Neuron. 100:684699.e6.

495 Aoki C, Venkatesan C, Go CG, Forman R, Kurose H. 1998. Cellular and subcellular sites for noradrenergic action in the monkey dorsolateral prefrontal cortex as revealed by the immunocytochemical localization of noradrenergic receptors and axons. Cereb Cortex. 8:269-277.

Aston-Jones G, Bloom F. 1981a. Nonrepinephrine-containing locus coeruleus neurons in behaving rats exhibit pronounced responses to non-noxious environmental stimuli. J Neurosci. 1:887-900.

Aston-Jones G, Bloom FE. 1981b. Activity of norepinephrine-containing locus coeruleus neurons in behaving rats anticipates fluctuations in the sleep-waking cycle. J Neurosci. 1:876-886.

Aston-Jones G, Cohen JD. 2005. An Integrative Theory of Locus CoeruleusNorepinephrine Function: Adaptive Gain and Optimal Performance. Annu Rev Neurosci. 28:403-450.

Barth AMI, Vizi ES, Zelles T, Lendvai B. 2008. a 2 -Adrenergic Receptors Modify Dendritic Spike Generation Via HCN Channels in the Prefrontal Cortex . J Neurophysiol. 99:394-401.

Berridge CW, Waterhouse BD. 2003. The locus coeruleus-noradrenergic system: modulation of behavioral state and state-dependent cognitive processes. Brain Res Rev. 42:33-84.

Breton-Provencher V, Sur M. 2018. Active control of arousal by a locus coeruleus GABAergic circuit SUPPLEMENTARY INFORMATION.

Carter ME, Yizhar O, Chikahisa S, Nguyen H, Adamantidis A, Nishino S, Deisseroth K, De Lecea L. 2010. Tuning arousal with optogenetic modulation of locus coeruleus neurons. Nat Neurosci. 13:1526-1533. 
Chandler DJ, Gao W-J, Waterhouse BD. 2014. Heterogeneous organization of the locus coeruleus projections to prefrontal and motor cortices. Proc Natl Acad Sci. 111:6816-6821.

Chen T-W, Wardill TJ, Sun Y, Pulver SR, Renninger SL, Baohan A, Schreiter ER, Kerr R a, Orger MB, Jayaraman V, Looger LL, Svoboda K, Kim DS. 2013. Ultrasensitive fluorescent proteins for imaging neuronal activity. Nature. 499:295-300.

Cichon J, Gan W-B. 2015. Branch-specific dendritic Ca2+ spikes cause persistent synaptic plasticity. Nature. 520:180-185.

Dana H, Mohar B, Sun Y, Narayan S, Gordus A, Hasseman JP, Tsegaye G, Holt GT, Hu A, Walpita D, Patel R, Macklin JJ, Bargmann Cl, Ahrens MB, Schreiter ER, Jayaraman V, Looger LL, Svoboda K, Kim DS. 2016. Sensitive red protein calcium indicators for imaging neural activity. Elife. 5:1-24.

Dembrow NC, Chitwood RA, Johnston D. 2010. Projection-Specific Neuromodulation of Medial Prefrontal Cortex Neurons. J Neurosci. 30:16922-16937.

Ding F, O'Donnell J, Thrane AS, Zeppenfeld D, Kang H, Xie L, Wang F, Nedergaard M. 2013. a1-Adrenergic receptors mediate coordinated Ca2+ signaling of cortical astrocytes in awake, behaving mice. Cell Calcium. 54:387-394.

Dunn M, Henke A, Clark S, Kovalyova Y, Kempadoo KA, Karpowicz RJ, Kandel ER, Sulzer D, Sames D. 2018. Designing a norepinephrine optical tracer for imaging individual noradrenergic synapses and their activity in vivo. Nat Commun. 9:2838.

Einhäuser W, Stout J, Koch C, Carter O. 2008. Pupil dilation reflects perceptual selection and predicts subsequent stability in perceptual rivalry. Proc Natl Acad Sci U S A. 105:1704-1709.

Feng J, Zhang C, Lischinsky JE, Jing M, Zhou J, Wang H, Zhang Y, Dong A, Wu Z, Wu H, Chen W, Zhang P, Zou J, Hires SA, Zhu JJ, Cui G, Lin D, Du J, Li Y. 2019. A Genetically Encoded Fluorescent Sensor for Rapid and Specific In Vivo Detection of Norepinephrine. Neuron. 102:745-761.e8.

Florin-Lechner SM, Druhan JP, Aston-Jones G, Valentino RJ. 1996. Enhanced norepinephrine release in prefrontal cortex with burst stimulation of the locus coeruleus. Brain Res. 742:89-97. 
Garcia-Junco-Clemente P, Tring E, Ringach DL, Trachtenberg JT. 2019. State-

Dependent Subnetworks of Parvalbumin-Expressing Interneurons in Neocortex. Cell Rep. 26:2282-2288.e3.

Glennon E, Carcea I, Martins ARO, Multani J, Shehu I, Svirsky MA, Froemke RC. 2019. Locus coeruleus activation accelerates perceptual learning. Brain Res. 1709:39-49.

Herkenham M. 1987. Mismatches between neurotransmitter and receptor localizations in brain: observations and implications. Neuroscience. 23:1-38.

Hervé-Minvielle A, Sara SJ. 1995. Rapid habituation of auditory responses of locus coeruleus cells in anaesthetized and awake rats. Neuroreport. 6:1363-1368.

Joshi S, Li Y, Kalwani RM, Gold JI. 2016. Relationships between Pupil Diameter and Neuronal Activity in the Locus Coeruleus, Colliculi, and Cingulate Cortex. Neuron. 89:221-234.

Kerr JND, de Kock CPJ, Greenberg DS, Bruno RM, Sakmann B, Helmchen F. 2007. Spatial Organization of Neuronal Population Responses in Layer 2/3 of Rat Barrel Cortex. J Neurosci. 27:13316-13328.

Kim J-H, Jung A-H, Jeong D, Choi I, Kim K, Shin S, Kim SJ, Lee S-H. 2016.

Selectivity of Neuromodulatory Projections from the Basal Forebrain and Locus Ceruleus to Primary Sensory Cortices. J Neurosci. 36:5314-5327.

570 Labarrera C, Deitcher Y, Dudai A, Weiner B, Kaduri Amichai A, Zylbermann N, London M. 2018. Adrenergic Modulation Regulates the Dendritic Excitability of Layer 5 Pyramidal Neurons In Vivo. Cell Rep. 23:1034-1044.

Lindeberg J, Usoskin D, Bengtsson H, Gustafsson A, Kylberg A, Söderström S, Ebendal T. 2004. Transgenic expression of Cre recombinase from the tyrosine hydroxylase locus. genesis. 40:67-73.

Lorang D, Amara SG, Simerly RB. 1994. Cell-type-specific expression of catecholamine transporters in the rat brain. J Neurosci. 14:4903-4914.

Manita S, Suzuki T, Homma C, Matsumoto T, Odagawa M, Yamada K, Ota K, Matsubara C, Inutsuka A, Sato M, Ohkura M, Yamanaka A, Yanagawa Y, Nakai J, Hayashi Y, Larkum ME, Murayama M. 2015. A Top-Down Cortical Circuit for Accurate Sensory Perception. Neuron. 86:1304-1316.

Manunta Y, Edeline J-M. 2004. Noradrenergic Induction of Selective Plasticity in the 
Frequency Tuning of Auditory Cortex Neurons. J Neurophysiol. 92:1445-1463.

Martins ARO, Froemke RC. 2015. Coordinated forms of noradrenergic plasticity in the locus coeruleus and primary auditory cortex. Nat Neurosci. 18:1483-1492.

McGinley MJ, David S V., McCormick DA. 2015. Cortical Membrane Potential Signature of Optimal States for Sensory Signal Detection. Neuron. 87:179-192.

Miyamoto D, Hirai D, Fung CCA, Inutsuka A, Odagawa M, Suzuki T, Boehringer R, Adaikkan C, Matsubara C, Matsuki N, Fukai T, McHugh TJ, Yamanaka A, 590 Murayama M. 2016. Top-down cortical input during NREM sleep consolidates perceptual memory. Science. 352:1315-1318.

Muller A, Joseph V, Slesinger PA, Kleinfeld D. 2014. Cell-based reporters reveal in vivo dynamics of dopamine and norepinephrine release in murine cortex. Nat Methods. 11:1245-1252.

595 Nicholas AP, Pieribone VA, Hökfelt T. 1993. Cellular localization of messenger RNA for beta-1 and beta-2 adrenergic receptors in rat brain: An in situ hybridization study. Neuroscience. 56:1023-1039.

Niell CM, Stryker MP. 2008. Highly selective receptive fields in mouse visual cortex. J Neurosci. 28:7520-7536.

600 Park J, Bhimani R V., Bass CE. 2018. Review_In Vivo Electrochemical Measurements of Norepinephrine in the Brain: Current Status and Remaining Challenges. J Electrochem Soc. 165:G3051-G3056.

Petersen CCH, Hahn TTG, Mehta M, Grinvald A, Sakmann B. 2003. Interaction of sensory responses with spontaneous depolarization in layer $2 / 3$ barrel cortex. Proc Natl Acad Sci. 100:13638-13643.

Phillips WA, Bachmann T, Storm JF. 2018. Apical Function in Neocortical Pyramidal Cells: A Common Pathway by Which General Anesthetics Can Affect Mental State. Front Neural Circuits. 12:50.

Phillips WA, Larkum ME, Harley CW, Silverstein SM. 2016. The effects of arousal on apical amplification and conscious state. Neurosci Conscious. 2016:niw015.

Polack PO, Friedman J, Golshani P. 2013. Cellular mechanisms of brain statedependent gain modulation in visual cortex. Nat Neurosci. 16:1331-1339.

Ranganathan GN, Apostolides PF, Harnett MT, Xu NL, Druckmann S, Magee JC. 2018. Active dendritic integration and mixed neocortical network representations 
during an adaptive sensing behavior. Nat Neurosci. 21:1583-1590.

Reimer J, Froudarakis E, Cadwell CR, Yatsenko D, Denfield GH, Tolias AS. 2014.

Pupil Fluctuations Track Fast Switching of Cortical States during Quiet Wakefulness. Neuron. 84:355-362.

Reimer J, McGinley MJ, Liu Y, Rodenkirch C, Wang Q, McCormick DA, Tolias AS. 2016. Pupil fluctuations track rapid changes in adrenergic and cholinergic activity in cortex. Nat Commun. 7:13289.

Rho H-J, Kim J-H, Lee S-H. 2018. Function of Selective Neuromodulatory Projections in the Mammalian Cerebral Cortex: Comparison Between Cholinergic and Noradrenergic Systems. Front Neural Circuits. 12:1-13.

625 Rodenkirch C, Liu Y, Schriver BJ, Wang Q. 2019. Locus coeruleus activation enhances thalamic feature selectivity via norepinephrine regulation of intrathalamic circuit dynamics. Nat Neurosci. 22:120-133.

Sara SJ. 2009. The locus coeruleus and noradrenergic modulation of cognition. Nat Rev Neurosci. 10:211-223.

630 Sara SJ, Bouret S. 2012. Orienting and Reorienting: The Locus Coeruleus Mediates Cognition through Arousal. Neuron. 76:130-141.

Sara SJ, Segal M. 1991. Plasticity of sensory responses of locus coeruleus neurons in the behaving rat: Implications for cognition, Progress in Brain Research. Elsevier B.V.

635 Schwarz LA, Miyamichi K, Gao XJ, Beier KT, Weissbourd B, DeLoach KE, Ren J, Ibanes S, Malenka RC, Kremer EJ, Luo L. 2015. Viral-genetic tracing of the input-output organization of a central noradrenaline circuit. Nature. 524:88-92.

Swanson LW. 1976. The locus coeruleus: A cytoarchitectonic, golgi and immunohistochemical study in the albino rat. Brain Res. 110:39-56.

640 Takahashi N, Oertner TG, Hegemann P, Larkum ME. 2016. Active cortical dendrites modulate perception. Science (80- ). 354:1587-1590.

Varazzani C, San-Galli A, Gilardeau S, Bouret S. 2015. Noradrenaline and Dopamine Neurons in the Reward/Effort Trade-Off: A Direct Electrophysiological Comparison in Behaving Monkeys. J Neurosci. 35:7866-7877. 
a2A-Adrenoceptors Strengthen Working Memory Networks by Inhibiting cAMPHCN Channel Signaling in Prefrontal Cortex. Cell. 129:397-410.

Waterhouse BD, Chandler DJ. 2016. Heterogeneous organization and function of the central noradrenergic system. Brain Res. 1641:v-x.

Williams SR, Fletcher LN. 2019. A Dendritic Substrate for the Cholinergic Control of Neocortical Output Neurons. Neuron. 101:486-499.e4.

Xu N, Harnett MT, Williams SR, Huber D, O'Connor DH, Svoboda K, Magee JC, O'Connor DH, Svoboda K, Magee JC. 2012. Nonlinear dendritic integration of sensory and motor input during an active sensing task. Nature. 492:247-251. 